\title{
Pilot Tone Selection in OFDM Amplified-and-forward Relay System
}

\author{
Linfeng An, Ming Chen, Bingyang Wu \\ National Mobile Communication Research Laboratory \\ Southeast University \\ Nanjing, China \\ E-mail: ydlinfeng1026@126.com
}

\begin{abstract}
Channel estimation for OFDM amplify-and-forward relay system requires transmission of pilot tones in source node, and different pilot tones can be derived according to different channel estimation algorithms and OFDM scenarios. This paper derives two different pilot tones with different scenarios: 1) no virtual subcarrier is applied in OFDM system and LS algorithm is used in channel estimation; 2) virtual subcarriers are applied in OFDM system and RLS algorithm is used in channel estimation.
\end{abstract} $R L S$

Keywords-relay; OFDM; pilot tone; virtual subcarrier; LS ;

\section{INTRODUCTION}

Recently, there has been considerable interest in relay technique. A relay system can improve Spectrum utilization, promote signal quality of users in cellular edge, and increase system capacity [1]. Therefore, relay technique will be of crucial importance in future mobile systems.

In order to study OFDM amplify-and-forward system, the destination node needs to have the knowledge of channel information. to acquire accurate channel information, the pilot tone needs to be carefully designed.

\section{A. Two scenarios studied in this paper}

Different pilot tones can be derived according to different channel estimation algorithms and OFDM scenarios. In this paper, two OFDM amplify-and-forward relay channel pilot tones are studied according to two different algorithms and OFDM scenarios.

Suppose number of pilot and data subcarriers are $k_{\max }$, and $N$ points IFFT is operated in OFDM modulation, $N \geq k_{\text {max }}, N=2^{n}$, and $n$ is an integer. If $N>k_{\text {max }}$, some subcarriers are used to transmit pilot and data information, and the rest subcarriers are left unoccupied. The unoccupied subcarriers are named virtual subcarriers. If $k_{\max }=N$, all subcarriers are used to transmit data or pilot information, and there are no virtual subcarriers in the system.

The first scenario is that: no virtual subcarrier is applied in OFDM system, and LS algorithm [2]-[3] is used for channel estimation. Pilot tone based on scenario 1 in OFDM amplify-and-forward relay channel is studied at first. Similar work has been done in [4], and its analysis is based on traditional OFDM systems, which is quite helpful for the work in this paper.

If there are virtual subcarriers in OFDM system, applying LS algorithm will lead to ill-conditioned matrix, therefore, LS algorithm will be expired. To deal with this problem, RLS algorithm [6] is introduced. The second scenario is that: virtual subcarriers are applied in OFDM system, and RLS algorithm is used for channel estimation. Because LTE system has virtual subcarriers and the value of $N$ is relatively large, scenario 2 is more practical. Pilot tone based on scenario 2 in OFDM amplify-and-forward relay channel is studied at last.

\section{B. Organaization of this paper}

This paper is organized as followed. In section II, OFDM amplify-and-forward relay channel is modeled; pilot tone based on scenario 1 is studied in section III; pilot tone based on scenario 2 is studied in section IV simulation results are presented in section V; conclusion is drawn in section VI.

\section{OFDM AMPLIFY-AND-FORWARD RELAY CHANNEL MODEL}

\section{A. OFDM Amplify-and-forward relay systemr}

As shown in Fig. 1, an OFDM amplify-and-forward system is comprised of a source node $\mathrm{S}$, a relay node $\mathrm{R}$ and $\mathrm{a}$ destination node $\mathrm{D}$. The transmission can be divided into two slots, in the first slot, source node transmits the OFDM modulated signal to relay node; while in the second slot, relay node amplify and retransmits the signal to destination node.

\section{B. Channel model}

OFDM signal received in the first slot can be denoted as

$$
R[k]=S[k] H_{1}[k]+N_{1}[k], k=0,1,2 \cdots k_{\max }-1
$$

Where $k$ is the subcarrier number; $N$ is the subcarrier number one OFDM symbol possesses, $N=2^{n}$, and $n$ is positive integer; $R[k]$ is $k$ th subcarrier signal received by relay node; $S[k]$ is $k$ th subcarrier signal transmitted by source node; $H_{1}[k]$ is $k$ th subcarrier frequency response of source-relay channel, and $H_{1}[k]$ is a complex Gaussian random variable

Supported by National Science and Technology Key Special Projects (No 2010ZX03003-002 \& 2010ZX03003-004), National Nature Science

Foundation of China (No. 60972023), and Research Fund of National Mobile

Communications Research Laboratory (No. 2011A06), and UK-China

Science Bridge: R\&D on $4 \mathrm{G}$ wireless mobile communications. 
with the variance $K_{1} ; N_{1}[k]$ is the complex white Gaussian noise of $k$ th subcarrier of relay node, and its variance is $\sigma_{1}^{2}$.

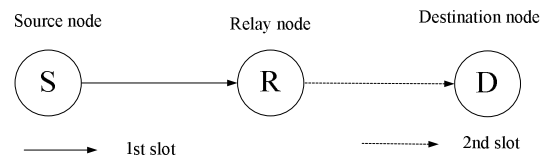

Figure 1. OFDM amplify-and-forward system

In the second slot, OFDM signal received at destination node can be denoted

$$
\begin{aligned}
D[k] & =G R[k] H_{2}[k]+N_{2}[k] \\
& =G S[k] H_{1}[k] H_{2}[k]+G H_{2}[k] N_{1}[k]+N_{2}[k]
\end{aligned}
$$

Where $D[k]$ is $k$ th subcarrier signal received at destination node; $H_{2}[k]$ is $k$ th subcarrier frequency response of relay-destination channel, and $H_{2}[k]$ is a complex Gaussian random variable with the variance $K_{2} ; N_{2}[k]$ is the complex white Gaussian noise of $k$ th subcarrier in destination node, and its variance is $\sigma_{2}^{2} ; G$ is the amplifying gain of the relay node.

Suppose subcarrier set $\left\{k_{1}, k_{2}, \cdots, k_{M}\right\}$ is the pilot tone used to transmit pilots. Pilot subcarriers received at destination node can be denoted in matrix form [4] as

$$
\mathbf{Y}_{P}=\mathbf{X}_{P} \mathbf{H}_{P}+\varepsilon_{P}=\mathbf{X}_{P} \mathbf{Q} h+\varepsilon_{P}
$$

Where, $\quad \mathbf{Y}_{P}=\left[D\left[k_{1}\right], D\left[k_{2}\right], \cdots, D\left[k_{M}\right]\right]^{\mathrm{T}}$, and $D\left[k_{i}\right]$ denotes $k_{i}$ th pilot subcarrier signals received at destination node; $\mathbf{X}_{P}=\operatorname{diag}\left(S\left[k_{1}\right], S\left[k_{2}\right], \cdots, S\left[k_{M}\right]\right)$, and $S\left[k_{i}\right]$ denotes pilot subcarrier signal transmitted by source node, and power of $S\left[k_{i}\right]$ is normalized to $\mathbf{1} ; \mathbf{h}$ denotes source-relay-destination time domain channel response, and $\mathbf{h}=\left[h_{1}, h_{2}, \cdots, h_{L}\right]^{\mathrm{T}}$, where $h_{i}$ denotes channel gain at the $i$ th tap, and $L$ is maximum length of channel, and $N / L$ is an integer; $\varepsilon_{P}=\left[\varepsilon_{1}, \varepsilon_{2}, \cdots \varepsilon_{M}\right]$ is the noise vector, and $i$ th element of the vector $\varepsilon_{P}$ is $\varepsilon_{i}=G H_{2}\left[k_{i}\right] N_{1}\left[k_{i}\right]+N_{2}\left[k_{i}\right]$, denoting noise in $k_{i}$ th pilot subcarrier signals.

$\mathbf{Q}$ is the FFT transformation matrix, it can be written as

$$
\mathbf{Q}=\left(\begin{array}{cccc}
1 & W_{N}^{k_{1}} & \ldots & W_{N}^{k_{1}(L-1)} \\
1 & W_{N}^{k_{2}} & \ldots & W_{N}^{k_{2}(L-1)} \\
\cdots & \ldots & \ldots & \ldots \\
1 & W_{N}^{k_{M}} & \ldots & W_{N}^{k_{M}(L-1)}
\end{array}\right)
$$

Where $W_{N}^{k_{i}}=\mathrm{e}^{-j \frac{2 \pi k_{i}}{N}}$.

$\mathbf{H}_{P}=\mathbf{Q h}=\left[G H_{1}\left[k_{1}\right] H_{2}\left[k_{1}\right], \cdots, G H_{1}\left[k_{M}\right] H_{2}\left[k_{M}\right]\right]^{\mathrm{T}}$, denoting frequency domain channel of pilot subcarriers.

\section{OPTIMUM PILOT TONES IN THE FIRST SCENARIO}

In the first scenario, no virtual subcarrier is applied in the system, and LS algorithm is used for channel estimation.

\section{A. OFDM Amplify-and-forward relay systemr}

For(3), $\mathbf{Y}_{P}$ is the observed value, $\mathbf{h}_{P}$ is a variable to be estimated and $\varepsilon_{P}$ is an unknown noise vector. Deriving from LS algorithm

$$
\begin{aligned}
h_{\mathrm{LS}} & =\left(\mathbf{Q}^{\mathrm{H}} \mathbf{X}_{P}^{\mathrm{H}} \mathbf{X}_{P} \mathbf{Q}\right)^{-1} \mathbf{Q}^{\mathrm{H}} \mathbf{X}_{P}^{\mathrm{H}} \mathbf{Y}_{P} \\
& =\left(\mathbf{Q}^{\mathrm{H}} \mathbf{Q}\right)^{-1} \mathbf{Q}^{\mathrm{H}} \mathbf{X}_{P}^{-1} \mathbf{Y}_{P}
\end{aligned}
$$

From the equation above, it is observed that the channel information is recovered as long as $\mathbf{Q}^{\mathrm{H}} \mathbf{Q}$ is invertible. According to [4], the minimum number of pilot subcarriers is used to restore the channel, that is $M=L$.As a result, set $\left\{k_{1}, k_{2}, \cdots, k_{L}\right\}$ should be the transmitted subcarrier set, and

$$
0 \leq k_{1} \leq k_{2} \leq \cdots \leq k_{L} \leq N-1
$$

matrix $\mathbf{Q}$ can be rewritten as

$$
\mathbf{Q}=\left(\begin{array}{cccc}
1 & W_{N}^{k_{1}} & \cdots & W_{N}^{k_{1}(L-1)} \\
1 & W_{N}^{k_{2}} & \cdots & W_{N}^{k_{2}(L-1)} \\
\cdots & \cdots & \cdots & \cdots \\
1 & W_{N}^{k_{L}} & \cdots & W_{N}^{k_{L}(L-1)}
\end{array}\right)
$$

In(5), $\quad \mathbf{X}_{P}^{-1} \mathbf{Y}_{P}=\left[\frac{D\left[k_{1}\right]}{S\left[k_{1}\right]}, \frac{D\left[k_{2}\right]}{S\left[k_{2}\right]}, \cdots, \frac{D\left[k_{L}\right]}{S\left[k_{L}\right]}\right]^{\mathrm{T}} \quad, \quad i$ th $\quad$ element denotes $k_{i}$ th pilot subcarrier frequency response, observing (2),it can be written as

$$
\begin{aligned}
\frac{D\left[k_{i}\right]}{S\left[k_{i}\right]} & =G H_{1}\left[k_{i}\right] H_{2}\left[k_{i}\right]+G \frac{H_{2}\left[k_{i}\right] N_{1}\left[k_{i}\right]}{S\left[k_{i}\right]}+\frac{N_{2}\left[k_{i}\right]}{S\left[k_{i}\right]} \\
& =H\left[k_{i}\right]+G H_{2}\left[k_{i}\right] N_{1}^{\prime}\left[k_{i}\right]+N_{2}^{\prime}\left[k_{i}\right]
\end{aligned}
$$


Where $k_{i} \in\left\{k_{1}, k_{2}, \cdots, k_{L}\right\} ; H\left[k_{i}\right]=G H_{1}\left[k_{i}\right] H_{2}\left[k_{i}\right]$ is the real source-relay-destination channel frequency response; $N_{1}^{\prime}\left[k_{i}\right]=\frac{N_{1}\left[k_{i}\right]}{S\left[k_{i}\right]}$, because power of each pilot subcarrier is normalized to $1, N_{1}^{\prime}[k]$ is a complex Gaussian random with mean-square-error $\sigma_{1}^{2} ; N_{2}^{\prime}\left[k_{i}\right]=\frac{N_{2}\left[k_{i}\right]}{S\left[k_{i}\right]}$, and $N_{2}^{\prime}\left[k_{i}\right]$ is a complex Gaussian random with mean-square-error $\sigma_{2}^{2}$.

And $\mathbf{X}_{P}^{-1} \mathbf{Y}_{P}$ can be rewritten as

$$
\mathbf{X}_{P}^{-1} \mathbf{Y}_{P}=\mathbf{H}_{P}+\mathbf{N}_{P}
$$

Where frequency noise vector $\mathbf{N}_{P}$ is

$$
\begin{aligned}
\mathbf{N}_{P} & =\left[N_{1}, \cdots, N_{M}\right]^{\mathrm{T}} \\
& =\left[H_{2}\left[k_{1}\right] N_{1}^{\prime}\left[k_{1}\right]+N_{2}^{\prime}\left[k_{1}\right], \cdots, H_{2}\left[k_{M}\right] N_{1}^{\prime}\left[k_{M}\right]+N_{2}^{\prime}\left[k_{M}\right]\right]^{\mathrm{T}}
\end{aligned}
$$

$N_{i}$ represents source-relay-destination equivalent noise at $i$ th pilot subcarrier .

Substitute (9) to (5),

$$
h_{\mathrm{LS}}=\mathbf{Q}^{-1} \mathbf{H}_{P}+\mathbf{Q}^{-1} \mathbf{N}_{P}=\mathbf{h}+\mathbf{Q}^{-1} \mathbf{N}_{P}
$$

\section{B. Optimum pilot tones}

Observing(10), if there is no noise at relay node and destination node, any $L$ pilot subcarriers can recover the channel information completely. Pilot tones determine the mean square error of the estimated channel when the noise exists.

Let pilot subcarrier set $\left\{k_{1}, k_{2}, \cdots, k_{L}\right\}$ be used to transmit pilots, the mean-square-error of the estimated time domain channel is

$$
\begin{aligned}
& E\left\{\left\|\mathbf{h}-\mathbf{h}_{\mathrm{LS}}\right\|^{2}\right\} \\
& =E\left\{\operatorname{tr}\left\{\mathbf{Q}^{-1} \mathbf{N}_{P}\left(\mathbf{Q}^{-1} \mathbf{N}_{P}\right)^{\mathrm{H}}\right\}\right\} \\
& =\operatorname{tr}\left\{\mathbf{Q}^{-1}\left[\begin{array}{cccc}
E\left[N_{1} N_{1}^{*}\right] & E\left[N_{1} N_{2}^{*}\right] & \cdots & E\left[N_{1} N_{L}^{*}\right] \\
E\left[N_{2} N_{1}^{*}\right] & E\left[N_{2} N_{2}^{*}\right] & \cdots & E\left[N_{2} N_{L}^{*}\right] \\
\cdots & \cdots & \cdots & \cdots \\
E\left[N_{L} N_{1}^{*}\right] & E\left[N_{L} N_{2}^{*}\right] & \cdots & E\left[N_{L} N_{L}^{*}\right]
\end{array}\right]\left(\mathbf{Q}^{-1}\right)^{\mathrm{H}}\right\}
\end{aligned}
$$

Where

$$
\begin{aligned}
& E\left\{N_{i} N_{j}^{*}\right\} \\
& =E\left\{\left(G H_{2}\left[k_{i}\right] N_{1}^{\prime}\left[k_{i}\right]+N_{2}^{\prime}\left[k_{i}\right]\right)\left(G H_{2}\left[k_{j}\right] N_{1}^{\prime}\left[k_{j}\right]+N_{2}^{\prime}\left[k_{j}\right]\right)^{*}\right\} \\
& =\left\{\begin{array}{l}
E\left\{G^{2} H_{2}\left[k_{i}\right] H_{2}^{*}\left[k_{i}\right]\right\} E\left\{N_{1}^{\prime}\left[k_{i}\right] N_{1}^{\prime *}\left[k_{i}\right]\right\}+E\left\{N_{2}^{\prime}\left[k_{i}\right] N_{2}^{\prime *}\left[k_{i}\right]\right\}, i= \\
0 \quad, i \neq j
\end{array}\right. \\
& = \begin{cases}K_{2} G^{2} \sigma_{1}^{2}+\sigma_{2}^{2}, i=j \\
0 \quad, i \neq j\end{cases}
\end{aligned}
$$

Substitute (11) to (10)

$$
E\left\{\|\mathbf{h}-\tilde{\mathbf{h}}\|^{2}\right\}=\left(K_{2} G^{2} \sigma_{1}^{2}+\sigma_{2}^{2}\right) \operatorname{tr}\left\{\left(\mathbf{Q}^{\mathrm{H}} \mathbf{Q}\right)^{-1}\right\}
$$

Theorem: when $E\left\{\|\mathbf{h}-\tilde{\mathbf{h}}\|^{2}\right\}$ get the minimum value, the pilot subcarrier set should be

$$
\left\{i, i+\frac{N}{L}, \cdots, i+\frac{(L-1) N}{L}\right\}, i=0,1,2 \cdots, \frac{N}{L}-1
$$

Proof:

Let eigenvalues of $\left(\mathbf{Q}^{\mathrm{H}} \mathbf{Q}\right)^{-1}$ be $\left\{\lambda_{p}, p=1,2 \cdots, L\right\}$, then eigenvalues of $\mathbf{Q}^{\mathrm{H}} \mathbf{Q}$ is $\left\{1 / \lambda_{p}, p=1,2 \cdots, L\right\}$. Noticing that $\operatorname{tr}\left\{\mathbf{Q}^{\mathrm{H}} \mathbf{Q}\right\}=L^{2}, \quad$ the following equation can be obtained.

$$
\left\{\begin{array}{l}
\min \sum_{i=1}^{L} \lambda_{p} \\
\text { s.t. } \sum_{i=1}^{L} \frac{1}{\lambda_{p}}=L^{2}
\end{array}\right.
$$

$\left(\mathbf{Q}^{\mathrm{H}} \mathbf{Q}\right)^{-1}$ is nonnegative definite matrix, the eigenvalues are also nonnegative. Iff all the eigenvalues are the same, sum of eigenvalues are the minimum.

Because $\operatorname{tr}\left\{\mathbf{Q}^{\mathrm{H}} \mathbf{Q}\right\}=L^{2} / N$, and all eigenvalues are the same, the following can be obtained

$$
\mathbf{Q}^{\mathrm{H}} \mathbf{Q}=L \mathbf{U}^{\mathrm{H}} \mathbf{I} \mathbf{U}=L \mathbf{I}
$$

Where $\mathbf{U}$ is unitary matrix.

Notice that any column vector of $\mathbf{Q}$ is orthogonal to another column vector, inner product of $i$ th column and $j$ th column can be denoted as 


$$
\sum_{l=0}^{L-1} \mathrm{e}^{-\mathrm{j} \frac{2 \pi}{N}\left(k_{i}-k_{j}\right) l}=\frac{1-\mathrm{e}^{-\mathrm{j} \frac{2 \pi}{N}\left(k_{i}-k_{j}\right) L}}{1-\mathrm{e}^{-\mathrm{j} \frac{2 \pi}{N}\left(k_{i}-k_{j}\right)}}=0
$$

That is,

$$
\begin{gathered}
\mathrm{e}^{-\mathrm{j} \frac{2 \pi}{N}\left(k_{i}-k_{j}\right) L}=\mathrm{e}^{-\mathrm{j} 2 \pi \mathrm{M}} \\
\left(k_{i}-k_{j}\right)=M \frac{N}{L}, M=0,1,2, \cdots
\end{gathered}
$$

As a result of $0<\left(k_{i}-k_{j}\right)<N, M=0,1,2 \cdots, \frac{N}{L}-1$, that is, $\left\{i, i+\frac{N}{L}, \cdots, i+\frac{(L-1) N}{L}\right\}, i=0,1,2 \cdots, \frac{N}{L}-1$ is the only pilot tones satisfying equation(15).

The proof is done.

If no virtual subcarrier is applied in the system and LS algorithm is used for channel estimation, equal-spaced pilot subcarrier sets are the optimal pilot tones.

\section{OPTIMUM PILOT TONES IN THE SECOND SCENARIO}

\section{A. RLS alforithm}

$\mathbf{Q}$ is a ill-conditioned matrix when virtual subcarrier is applied, and the LS algorithm expires at this time. Therefore, RLS algorithm is introduced

$$
\begin{aligned}
h_{\mathrm{RLS}} & =\left(\mathbf{Q}^{\mathrm{H}} \mathbf{Q}+\alpha \mathbf{I}_{L}\right)^{-1} \mathbf{Q}^{\mathrm{H}} \mathbf{X}_{P}^{\mathrm{H}} \mathbf{Y}_{P} \\
& =\left(\mathbf{Q}^{\mathrm{H}} \mathbf{Q}+\alpha \mathbf{I}_{L}\right)^{-1} \mathbf{Q}^{\mathrm{H}} \mathbf{X}_{P}^{-1} \mathbf{Y}_{P} \\
& =\left(\mathbf{Q}^{\mathrm{H}} \mathbf{Q}+\alpha \mathbf{I}_{L}\right)^{-1} \mathbf{Q}^{\mathrm{H}}\left(\mathbf{H}_{P}+\mathbf{N}_{P}\right)
\end{aligned}
$$

Where $\alpha$ is a constant similar to noise vector.

\section{B. freqeuncy channel MSE and optimaziation problem}

Time domain channel obtained in(20) possess the channel information of all subcarriers, including virtual subcarriers. Because we just concern about data and pilot subcarriers, frequency channel of estimated data and pilot subcarriers is used to measure the performance of different pilot tones.

Frequency channel of data and pilot subcarriers is denoted as

$$
\begin{aligned}
\mathbf{H}_{\mathrm{RLS}} & =\mathbf{F}\left(\mathbf{Q}^{\mathrm{H}} \mathbf{Q}+\alpha \mathbf{I}_{L}\right)^{-1} \mathbf{Q}^{\mathrm{H}}\left(\mathbf{H}_{P}+\mathbf{N}_{P}\right) \\
& =\mathbf{H}+\left[\mathbf{F}\left(\mathbf{Q}^{\mathrm{H}} \mathbf{Q}+\alpha \mathbf{I}_{L}\right)^{-1} \mathbf{Q}^{\mathrm{H}}-\mathbf{F} \mathbf{Q}^{-1}\right] \mathbf{H}_{P} \\
& +\mathbf{F}\left(\mathbf{Q}^{\mathrm{H}} \mathbf{Q}+\alpha \mathbf{I}_{L}\right)^{-1} \mathbf{Q}^{\mathrm{H}} \mathbf{N}_{P}
\end{aligned}
$$

Where, $\mathbf{H}$ is the real frequency channel of data and pilot subcarriers, and FFT matrix $\mathbf{F}$ is

$$
\mathbf{F}=\left[\begin{array}{cccc}
1 & W_{N}^{1} & \cdots & W_{N}^{L-1} \\
1 & W_{N}^{2} & \cdots & W_{N}^{2(L-1)} \\
\cdots & \cdots & \cdots & \cdots \\
1 & W_{N}^{k_{\max }} & \cdots & W_{N}^{k_{\max }(L-1)}
\end{array}\right]
$$

$\operatorname{In}(21)$, the second term represents error introduced by the algorithm itself, that is, even if there is no noise, this error still take place. But if $\alpha$ is carefully chosen, this error is quite limited. The third term represents error introduced by noise.

When SNR is small and $\alpha$ is carefully chosen, the second term can be neglected. Several value of $\alpha$ is chosen, and when $\alpha=0.01$, better simulation performance can be obtained.

The mean-square-error of the third term can be denoted as

$$
\begin{aligned}
& E\left\{\left\|\left(\mathbf{Q}^{\mathrm{H}} \mathbf{Q}+\alpha \mathbf{I}_{L}\right)^{-1} \mathbf{Q}^{\mathrm{H}} \mathbf{N}_{P}\right\|^{2}\right\} \\
& =E\left\{\operatorname{tr}\left\{\mathbf{F}\left(\mathbf{Q}^{\mathrm{H}} \mathbf{Q}+\alpha \mathbf{I}_{L}\right)^{-1} \mathbf{Q}^{\mathrm{H}} \mathbf{N}\left(\mathbf{F}\left(\mathbf{Q}^{\mathrm{H}} \mathbf{Q}+\alpha \mathbf{I}_{L}\right)^{-1} \mathbf{Q}^{\mathrm{H}} \mathbf{N}\right)^{\mathrm{H}}\right\}\right\} \\
& =\left(K_{2} G^{2} \sigma_{1}^{2}+\sigma_{2}^{2}\right) \operatorname{tr}\left\{\mathbf{F}\left(\mathbf{Q}^{\mathrm{H}} \mathbf{Q}+\alpha \mathbf{I}_{L}\right)^{-1} \mathbf{Q}^{\mathrm{H}} \mathbf{Q}\left(\left(\mathbf{Q}^{\mathrm{H}} \mathbf{Q}+\alpha \mathbf{I}_{L}\right)^{-1}\right)^{\mathrm{H}} \mathbf{F}^{\mathrm{H}}\right\}
\end{aligned}
$$

Optimization problem can be denoted as

$$
\left\{\begin{array}{l}
\arg \min _{\left\{k_{1}, k_{2}, \cdots, k_{L}\right\}} \operatorname{tr}\left\{\mathbf{F}\left(\mathbf{Q}^{\mathrm{H}} \mathbf{Q}+\alpha \mathbf{I}_{L}\right)^{-1}\left(\left(\mathbf{Q}^{\mathrm{H}} \mathbf{Q}+\alpha \mathbf{I}_{L}\right)^{-1}\right)^{\mathrm{H}} \mathbf{F}^{\mathrm{H}}\right\} \\
\text { s.t. } 0 \leq k_{1} \leq k_{2} \leq \cdots \leq k_{L} \leq k_{\max }<N-1
\end{array}\right.
$$

\section{Searched results}

Genetic algorithm [7] is used to solve the optimization problem expressed in (24). The following table gives the searched results.

TABLE I. SEARCHED OPTIMUM PILOT TONES IN SCENARIO 3

\begin{tabular}{|c|c|c|c|}
\hline$N$ & $k_{\max }$ & $L$ & \multicolumn{1}{c|}{ searched pilot tones } \\
\hline \multirow{2}{*}{128} & \multirow{2}{*}{72} & \multirow{2}{*}{18} & $\begin{array}{l}{[0,3,10,13,26,31,39,40,41,} \\
42,43,44,45,46,51,59,66,71]\end{array}$ \\
\hline
\end{tabular}

Because the second term in (21) is neglected in formulizing the problem, and genetic algorithm may just get the suboptimal answer, the searched result is not necessarily optimum. But as shown by the simulated results in the next section, the obtained results still outperforms most of other pilot tones including equal-spaced pilot tone used in 3GPP protocols. Its performance is shown in the next section. 


\section{SIMULATION RESULTS}

\section{A. simualted results of the first scenario}

We consider a typical case in simulation, where one OFDM symbol has 128 subcarriers and tap number of source-relay-destination time domain channel is 8 . The simulated pilot tones are the presented equal-spaced pilot tones and other two pilot tones, while the "other two pilot tones" are randomly chosen to compare with theoretically optimum pilot tone. The pilot tones are specified in Fig 2.

The simulated results are shown in Fig 2, in the figure, $\mathrm{x}$-axis is the ratio of pilot power and total noise, while $\mathrm{y}$-axis is mean-square error of estimated source-relay-destination equivalent channel in time domain. Results prove that the presented equal-spaced pilot tone is the optimal pilot tones.

\section{$B$. simualted results of the second scenario}

A typical case is considered in simulation, where one OFDM symbol has 128 subcarriers. 54 subcarriers are used to transmit data, 18 subcarriers are used to transmit pilot, and the left subcarriers are unoccupied. Tap number of source-relay-destination time domain channel is 18 . The constant $\alpha=0.01$. The simulated pilot tones are the searched pilot tones, equal-spaced pilot tones and other two randomly chosen pilot tones. The pilot tone used in simulation is specified in TABLE II.

The simulated results is shown in Fig 3, in the figure, $\mathrm{x}$-axis is the ratio of pilot power and total noise, while $\mathrm{y}$-axis is normalized mean-square error of source-relay-destination frequency channel. Results prove that the searched pilot tone has better performance than other proposed pilot tones, and the performance of searched pilot tone is $6-7 \mathrm{~dB}$ better than the equal-spaced pilot tone that is currently used in 3GPP protocol.

TABLE II. PILOT TONE USED IN SIMULATION OF SCENARIO 2

\begin{tabular}{|c|l|}
\hline $\begin{array}{c}\text { searched pilot } \\
\text { tone }\end{array}$ & $\begin{array}{l}{[0,3,10,13,26,31,39,40,41,} \\
42,43,44,45,46,51,59,66,71]\end{array}$ \\
\hline $\begin{array}{c}\text { equal-spaced } \\
\text { pilot tone }\end{array}$ & {$[0,4,8,12,16,20,24,28,32,36$,} \\
other pilot & {$[1,3,5,7,4,26,27,29,40,47,50$,} \\
tone1 & $51,53,55,57,58,59,60,69]$ \\
\hline other pilot & {$[1,5,8,14,17,27,39,40,41,42$,} \\
tone2 & $43,44,45,46,50,56,66,69]$ \\
\hline
\end{tabular}

\section{CONCLUSION}

This paper answers the question of what pilot tone has better performance in OFDM amplify-and-forward system. Two different scenarios and their pilot tones are discussed, and each scenario has its own pilot tones. At last, the simulation results prove the superiority of presented pilot tones.

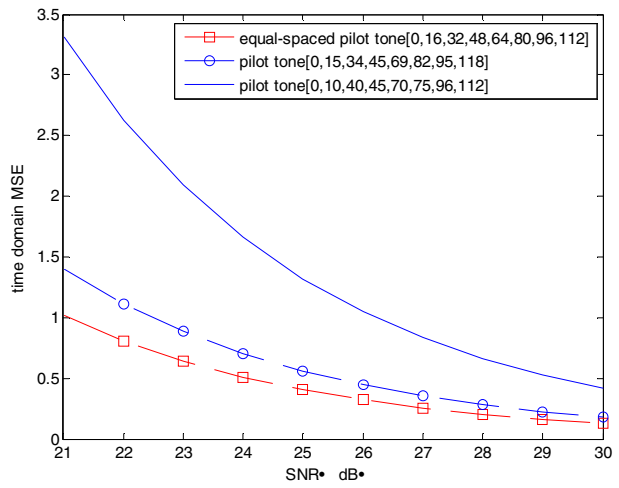

Figure 2. performance of pilot tones in scenario 1

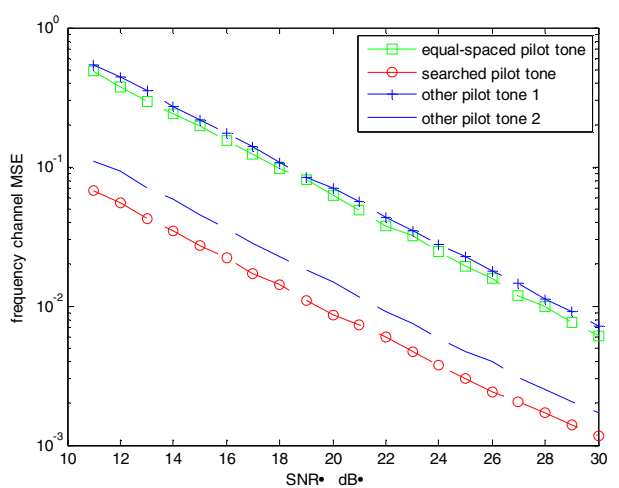

Figure 3. performance of pilot tones in scenario 2

\section{REFERENCES}

[1] C. Politis, T. Oda, S. Dixit, etal "Cooperative networks for the future wireless world”, IEEE Commun. Mag., Sep. 2004, Vol. 42, No. 9, pp: 70-79.

[2] Y. Li, "Pilot-symbol-aided channel estimation for OFDM in wireless systems, " IEEE Trans. Veh. Technol., vol. 49, no. 4, pp. 1207-1215, Jul. 2000

[3] M. H, C, Wei, "channel estimation for OFDM systems based on comb-type pilot arrangement in frequency selective fading channels", IEEE Trans. On Consumer Electronics, Feb. 1998, Vol. 44, No. 1, pp:217-225.

[4] R. Negl, J. Cioffi. "Pilot Tone Selection for Channel Estimation in a Mobile OFDM System", IEEE Trans. On Consumer Electronics, Aug,1998, pp:1122-1128.

[5] 3GPP TS 36.211 V9.1.0 "Evolved Universal Terrestrial Radio Acdess(E-UTRA); Physical channels and modulation".

[6] S. Omar, Andrea Ancora, "Performace analysis of general pilot-aided linear channel estimation in LTE OFDMA systems with application to simplified MMSE schemes", IEEE 19 th International symposium, Sep. 2008 , pp:1-6.

[7] D. Wang, J. Wang, H. Wang, "Intelligent optimization methods", Higher educational press, 2006 